\title{
Negative Regulation of Lymphocyte Activation
}

National Cancer Institute

\section{Source}

National Cancer Institute. Negative Regulation of Lymphocyte Activation. NCI

Thesaurus. Code C40846.

Lymphocyte Suppression involves interference with, or restraint of, the production and activity of lymphocytes. Formed in lymphoid tissue, lymphocytes are white blood cells that can generally be classified as either T- or B-cells. T-lymphocytes provide cellmediated immunity, while B-lymphocytes produce antibodies that provide humoral immunity. Suppression decreases the ability of lymphocytes to mount an immune response to invading substances. 